\title{
Low-energy collective electronic excitations in Pd metal
}

\author{
V. M. Silkin, ${ }^{1,2,3,4}$ I. P. Chernov, ${ }^{4}$ Yu. M. Koroteev, ${ }^{2,4,5}$ and E. V. Chulkov ${ }^{1,2}$ \\ ${ }^{1}$ Depto. de Física de Materiales and Centro Mixto CSIC-UPV/EHU, Facultad de Ciencias Químicas, Universidad del País Vasco, \\ Apdo. 1072, 20080 San Sebastián, Basque Country, Spain \\ ${ }^{2}$ Donostia International Physics Center (DIPC), P. de Manuel Lardizabal, 4, 20018 San Sebastián, Basque Country, Spain \\ ${ }^{3}$ IKERBASQUE, Basque Foundation for Science, 48011 Bilbao, Spain \\ ${ }^{4}$ Tomsk Polytechnical University, pr. Lenina 30, 634050 Tomsk, Russia \\ ${ }^{5}$ Institute of Strength Physics and Materials Science, Russian Academy of Sciences, pr. Academicheskii 2/1, 634021 Tomsk, Russia \\ (Received 17 August 2009; revised manuscript received 17 November 2009; published 21 December 2009)
}

\begin{abstract}
A theoretical study of collective electronic excitations in $\mathrm{Pd}$ at low-energy (from 0 to $\sim 3 \mathrm{eV}$ ) domain is reported. The calculations were performed with full inclusion of the electron band structure obtained within self-consistent pseudopotential approach. We show that the presence in Pd of two kinds of carriers (in $s-p$ and $d$ bands) at the Fermi level produces dramatic modification of the excitation spectra in this energy range in comparison with free-electron-like model prediction. In particular, at small momenta a peculiar plasmon mode with characteristic sound-like dispersion-an acoustic plasmon-is predicted to exist in this material. This mode has strong directional dependence on the momentum transfer: whereas for momenta along the $\langle 100\rangle$ and $\langle 111\rangle$ symmetry directions it arises as a single mode up to $\sim 1 \mathrm{eV}$, along the $\langle 110\rangle$ direction two acoustic modes (one of which disperses up to $\sim 2.5 \mathrm{eV}$ ) with different slope exist. As in many metallic systems, e.g., in transition metals, there are energy bands with large difference in the Fermi velocities, we expect that the existence of such plasmon mode must be a rather general phenomenon. Additionally, present calculations reveal other well-defined features in the energy-loss spectra in this low-energy range due to numerous interband transitions.
\end{abstract}

DOI: 10.1103/PhysRevB.80.245114

PACS number(s): 71.20.Be, 71.45.Gm, 72.15.Nj

\section{INTRODUCTION}

Single-particle electron-hole and collective electronic excitations in solids have been investigated for many years on the basis of the momentum- and frequency-dependent dynamical electron-density response. ${ }^{1}$ Basic knowledge on it was gained from a study of a free-electron gas (FEG) model in which an electron valence density of real solids is represented by an average electron density, characterized by an interelectron distance $-r_{s}$ parameter. ${ }^{2}$ For years this model was used in many studies, e.g., in order to obtain information on the dynamical exchange-correlation effects. However, direct comparison of predictions of FEG theories with measurements of real solids was rather difficult. In particular, very important and frequently dominant effects in dynamical dielectric properties of solids are introduced by electron band structure which in real solids can substantially deviate from the FEG one. For instance, the presence of more than one energy band can be a source of numerous interband transitions presented in real solids. Sometimes these transitions produce only little effect on the excitation spectra, introducing, e.g., a finite width in measured plasmon peaks (which must be zero up to certain momenta according to the FEG models, i.e., plasmon would live once excited for ever). However, frequently these transitions produce dramatic impact on the dielectric and optical properties of solids. A wellknown examples of this are, e.g., appearance of an additional low-energy plasmon peaks in layered materials ${ }^{3-9}$ and carbon nanotubes, ${ }^{10-12}$ dramatic redshift of plasmon frequency in $\mathrm{Ag},{ }^{13-15}$ or a negative momentum dependence of plasmon peak in Cs. ${ }^{16,17}$

For three-dimensional (3D) solids the FEG predicts that for collective excitation of an electron system or a plasmon mode, there is some threshold which is determined by average valence density. As in real metals $r_{s}$ is in the 1-6 a.u. range, these frequencies normally arise in the $\sim 3-20 \mathrm{eV}$ interval. Hence these plasmons can not participate directly in low-energy dynamical processes occurring in an electron system in the vicinity of the Fermi surface. On the other hand, in the 1950s it was pointed out that once in an electron system more than one energy bands with different Fermi velocities cross the Fermi level there exists possibility of appearance of an additional low-energy mode with peculiar sound-like dispersion-acoustic plasmon (AP). At small momenta $q$ the energy $\omega_{\mathrm{AP}}$ of this mode disperses linearly with $q$ as $\omega_{\mathrm{AP}}=u_{\mathrm{AP}} \cdot q,{ }^{18}$ i.e., $\omega_{\mathrm{AP}}$ tends to zero as $q \rightarrow 0$. Here the coefficient $u_{\mathrm{AP}}$ is a group velocity of the acoustic plasmon and it is determined by the Fermi velocity of the carriers in the energy band with lower Fermi velocity, $u_{F}^{\text {slow }}$, being $u_{\mathrm{AP}} \approx u_{F}^{\text {slow }}$. A similar idea of existence of an acoustic-like mode was proposed in the 1960s for explanation of elevated temperatures for transition to the superconducting state, $T_{c}$, in transition metals, ${ }^{19-21}$ where two kinds of carriers in both slow ( $d$ bands) and fast ( $s-p$ bands) are usually presented. ${ }^{22}$ The idea of acoustic plasmon existence was regularly evoked for explanation of elevated $T_{c}$ in superconductors with unusually $T_{c}$ (Refs. 23-25) which can be explained by the fact that the presence of an acoustic plasmon might dramatically affect the low-energy dynamical screening properties of an electron system. Nonetheless, to the best of our knowledge, this kind of plasmons has been shown to exist in any bulk material neither experimentally nor by $a b$ initio calculations. Only very recently a detailed $a b$ initio calculation of dynamical momentum-dependent response demonstrated its possible existence in the $\mathrm{MgB}_{2}$ compound for the momentum trans- 
fers along the direction perpendicular to the basal hexagonal boron planes. ${ }^{26,27}$ Note that a different type of acoustic plasmon characteristic for layered conductors can be caused by the out-of-phase motion of carriers in the neighboring layers. This mode allows to explain superconductivity in these materials. ${ }^{28-31}$

In the present paper we demonstrate that the first kind of a low-energy plasmon with acoustic-like dispersion can exist in a real 3D metal where more than one energy band crosses the Fermi level. Here, in a particular case of Pd it is shown that this acoustic plasmon is a truly $3 \mathrm{D}$ phenomenon and exists in all three high-symmetry directions. ${ }^{32}$ Note that the excitation spectra of palladium have been a subject of intense study in the past. It was investigated extensively by energyloss spectroscopy ${ }^{33-38}$ and optical ${ }^{39,40}$ experiments and also by $a b$ initio calculations. ${ }^{41-47}$ Besides some spread in the reported data one can observe that dielectric properties in $\mathrm{Pd}$ are determined by a dominant bulk plasmon mode located in the 7-8 eV energy range and several other less-pronounced peaks in energy-loss spectra due to numerous interband transitions. ${ }^{38,48}$ At the same time we are not aware of any similar detailed study of excitation spectra of this metal which would be focused on the low-energy (less than $\sim 1 \mathrm{eV}$ ) region.

The outline of the paper is as follows. In Sec. II we present the details of the $a b$ initio calculation of dynamical momentum-dependent dielectric function in 3D solids. Description and discussion of the obtained results are given in Sec. III. Finally, the main conclusions are summarized in Sec. IV. Atomic units $\left(\hbar=e^{2}=m_{e}=1\right)$ are used throughout unless otherwise is stated.

\section{CALCULATION METHOD}

Within the first Born approximation the inelastic scattering cross section of $\mathrm{x}$-rays and electrons for momentum transfer $\mathbf{q}$ and energy $\omega$ transferred to the system is proportional to the dynamical structure factor $S(\mathbf{q}, \omega)$ of manyelectron system. $S(\mathbf{q}, \omega)$ is related by the fluctuation- dissipation theorem ${ }^{1}$ to the dielectric function $\epsilon\left(\mathbf{r}, \mathbf{r}^{\prime}, \omega\right)$ which, in general, is a nonlocal energy-dependent function. For a periodic solid $S(\mathbf{q}, \omega)$ is

$$
S(\mathbf{q}, \omega)=-\frac{\Omega q^{2}}{2 \pi} \operatorname{Im}\left[\epsilon_{\mathbf{G}=0, \mathbf{G}^{\prime}=0}^{-1}(\mathbf{q}, \omega)\right],
$$

where $\Omega$ represents the normalization volume, $\mathbf{G}$ 's are the reciprocal lattice vectors, and $\operatorname{Im}\left[\epsilon_{\mathbf{G}=0, \mathbf{G}^{\prime}=0}^{-1}(\mathbf{q}, \omega)\right]$ is the energy-loss function related to the Fourier coefficients of the density-response function $\chi_{\mathbf{G}, \mathbf{G}^{\prime}}(\mathbf{q}, \omega)$ through

$$
\epsilon_{\mathbf{G}, \mathbf{G}^{\prime}}^{-1}(\mathbf{q}, \omega)=\delta_{\mathbf{G}, \mathbf{G}^{\prime}}+v_{\mathbf{G}}(\mathbf{q}) \chi_{\mathbf{G}, \mathbf{G}^{\prime}}(\mathbf{q}, \omega) .
$$

Here $v_{\mathbf{G}}(\mathbf{q})=4 \pi /|\mathbf{q}+\mathbf{G}|^{2}$ is Fourier transform of the Coulomb potential. Within linear-response theory $\chi$ relates the electron density, $n^{\text {ind }}(\mathbf{r}, \omega)$ induced in the system, to an external perturbation potential, $V^{\text {ext }}(\mathbf{r}, \omega)$, through the equation

$$
n^{\text {ind }}(\mathbf{r}, \omega)=\int \mathrm{d} \mathbf{r}^{\prime} \chi\left(\mathbf{r}, \mathbf{r}^{\prime}, \omega\right) V^{\text {ext }}\left(\mathbf{r}^{\prime}, \omega\right) \text {. }
$$

In the framework of time-dependent density-functional theory, ${ }^{49,50} \chi\left(\mathbf{r}, \mathbf{r}^{\prime}, \omega\right)$ obeys the integral equation

$$
\begin{aligned}
\chi\left(\mathbf{r}, \mathbf{r}^{\prime} \omega\right)= & \chi^{0}\left(\mathbf{r}, \mathbf{r}^{\prime} \omega\right)+\int \mathrm{d} \mathbf{r}_{1} \int \mathrm{d} \mathbf{r}_{2} \chi^{0}\left(\mathbf{r}, \mathbf{r}_{1}, \omega\right)\left[v\left(\mathbf{r}_{1}-\mathbf{r}_{2}\right)\right. \\
& \left.+K^{\mathrm{xc}}\left(\mathbf{r}_{1}, \mathbf{r}_{2}, \omega\right)\right] \chi\left(\mathbf{r}_{2}, \mathbf{r}^{\prime}, \omega\right)
\end{aligned}
$$

where $\chi^{0}\left(\mathbf{r}, \mathbf{r}^{\prime}, \omega\right)$ is the density-response function for a noninteracting electron system, $v\left(\mathbf{r}-\mathbf{r}^{\prime}\right)$ is the bare Coulomb potential, and $K^{\mathrm{xc}}\left(\mathbf{r}, \mathbf{r}^{\prime}, \omega\right)$ accounts for dynamical exchangecorrelation effects. For a periodic crystal the integral equation (4) transforms in the reciprocal space into a matrix equation

$$
\begin{aligned}
\chi_{\mathbf{G}, \mathbf{G}^{\prime}}(\mathbf{q}, \omega)= & \chi_{\mathbf{G}, \mathbf{G}^{\prime}}^{0}(\mathbf{q}, \omega)+\sum_{\mathbf{G}_{1}} \sum_{\mathbf{G}_{2}} \chi_{\mathbf{G}_{\mathbf{G}} \mathbf{G}_{1}}^{0}(\mathbf{q}, \omega) \\
& \times\left[v_{\mathbf{G}_{1}}(\mathbf{q}) \delta_{\mathbf{G}_{1}, \mathbf{G}_{2}}+K_{\mathbf{G}_{1}, \mathbf{G}_{2}}^{\mathrm{xc}}(\mathbf{q}, \omega)\right] \chi_{\mathbf{G}_{2}, \mathbf{G}^{\prime}}(\mathbf{q}, \omega) .
\end{aligned}
$$

The Fourier coefficients $\chi_{\mathbf{G}, \mathbf{G}^{\prime}}^{0}(\mathbf{q}, \omega)$ have a form

$$
\chi_{\mathbf{G}, \mathbf{G}^{\prime}}^{0},(\mathbf{q}, \omega)=\frac{2}{\Omega} \sum_{\mathbf{k}}^{B Z} \sum_{n, n^{\prime}} \frac{\left(f_{n \mathbf{k}}-f_{n^{\prime} \mathbf{k}+\mathbf{q}}\right)\left\langle\psi_{n \mathbf{k}}\left|e^{-\mathrm{i}(\mathbf{q}+\mathbf{G}) \cdot \mathbf{r}}\right| \psi_{n^{\prime} \mathbf{k}+\mathbf{q}}\right\rangle\left\langle\psi_{n^{\prime} \mathbf{k}+\mathbf{q}}\left|e^{\mathrm{i}\left(\mathbf{q}+\mathbf{G}^{\prime}\right) \cdot \mathbf{r}}\right| \psi_{n \mathbf{k}}\right\rangle}{\varepsilon_{n \mathbf{k}}-\varepsilon_{n^{\prime} \mathbf{k}+\mathbf{q}}+\omega+\mathrm{i} \eta},
$$

where the factor of 2 accounts for spin, the sums over $n$ and $n^{\prime}$ run over the band structure for wave vectors $\mathbf{k}$ in the first Brillouin zone (BZ), $f_{n \mathbf{k}}$ is the Fermi distribution function, $\varepsilon_{n \mathbf{k}}$ and $\psi_{n \mathbf{k}}(\mathbf{r})$ are Bloch eigenvalues and eigenfunctions, respectively, of the Kohn-Sham Hamiltonian, and $\eta$ a positive infinitesimal. Numerical evaluation of $\chi_{\mathbf{G G}^{\prime}}^{0}(\mathbf{q}, \omega)$ matrix is the most time-demanding part of such calculations and one should put some finite value ${ }^{51}$ for $\eta$ which introduces an additional broadening in the calculated spectra. In practice, instead of direct use of Eq. (6) the matrix $\chi_{\mathbf{G}, \mathbf{G}^{\prime}}^{0}(\mathbf{q}, \omega)$ can be evaluated by performing calculations at imaginary ${ }^{52}$ or complex ${ }^{53}$ frequencies $\omega$ with subsequent analytical continuation to the real axis. An efficient approach based on the tetrahedron method was realized as well. ${ }^{54}$ Here we use an alternative route consisting in calculating, at the first step, of the spectral 
function matrix $S_{\mathbf{G}, \mathbf{G}^{\prime}}^{0}(\mathbf{q}, \omega)$ (Refs. 55 and 56) according to expression

$$
\begin{aligned}
S_{\mathbf{G}, \mathbf{G}^{\prime}}^{0}(\mathbf{q}, \omega)= & \frac{2}{\Omega} \sum_{\mathbf{k}}^{B Z} \sum_{n}^{\text {occ unocc }} \sum_{n^{\prime}}\left\langle\psi_{n \mathbf{k}}\left|e^{-\mathrm{i}(\mathbf{q}+\mathbf{G}) \cdot \mathbf{r}}\right| \psi_{n^{\prime} \mathbf{k}+\mathbf{q}}\right\rangle \\
& \times\left\langle\psi_{n^{\prime} \mathbf{k}+\mathbf{q}}\left|e^{\mathrm{i}\left(\mathbf{q}+\mathbf{G}^{\prime}\right) \cdot \mathbf{r}}\right| \psi_{n \mathbf{k}}\right\rangle \delta\left(\varepsilon_{n \mathbf{k}}-\varepsilon_{n^{\prime} \mathbf{k}+\mathbf{q}}+\omega\right),
\end{aligned}
$$

with subsequent evaluation of the imaginary part of $\chi_{\mathbf{G}, \mathbf{G}^{\prime}}^{0},(\mathbf{q}, \omega)$ via relation

$$
S_{\mathbf{G}, \mathbf{G}^{\prime}}^{0}(\mathbf{q}, \omega)=-\frac{1}{\pi} \operatorname{sgn}(\omega) \operatorname{Im}\left[\chi_{\mathbf{G}, \mathbf{G}^{\prime}}^{0}(\mathbf{q}, \omega)\right] .
$$

Here $\operatorname{sgn}(\omega)=1(-1)$ for $\omega>0(\omega<0)$. In continuation, the real part of the $\chi_{\mathbf{G}, \mathbf{G}^{\prime}}^{0}(\mathbf{q}, \omega)$ matrix elements is obtained from the corresponding imaginary part by the Hilbert transform.

Present calculations have been performed for fcc Pd with the use of experimental lattice parameters $a_{c}=7.3512$ a.u. The one-particle energies $\varepsilon_{n \mathbf{k}}$ and wave functions $\psi_{n \mathbf{k}}(\mathbf{r})$ were obtained as a self-consistent solution of the Kohn-Sham equations with the use of exchange-correlation potential in the form of Ref. 57. The electron-ions interaction was described by a nonlocal norm-conserving ionic pseudopotential. ${ }^{58}$ Wave functions $\psi_{n \mathbf{k}}(\mathbf{r})$ were expanded in plane waves up to a kinetic-energy cutoff of $70 \mathrm{Ry}$. Up to 50 reciprocal vectors $\mathbf{G}$ were included in the Fourier expansions of $\chi^{0}, \chi$, and $\epsilon$ matrices. Hence, the local-field effects ${ }^{59,60}$ were incorporated in the evaluation of the energy-loss function, $\operatorname{Im}\left[\epsilon_{\mathbf{G}=0, \mathbf{G}^{\prime}=0}^{-1}(\mathbf{q}, \omega)\right]$, through the inclusion of nondiagonal matrix elements of $\chi_{\mathbf{G}, \mathbf{G}^{\prime}}^{0}(\mathbf{q}, \omega)$ in matrix Eq. (5). In Eq. (7) the sum over $\mathbf{k}$ includes a $144 \times 144 \times 144$ sampling, that corresponds to 1492992 points in the BZ. The sums over $n$ and $n^{\prime}$ included energy bands up to energy of $50 \mathrm{eV}$ above the Fermi level. The $S_{\mathbf{G}, \mathbf{G}^{\prime}}^{0}(\mathbf{q}, \omega)$ matrices were calculated at the discrete mesh of energies ranging from 0 to 50 $\mathrm{eV}$ with a step of $0.01 \mathrm{eV}$. In the numerical calculations the $\delta$ function in Eq. (7) was replaced by a Gaussian with a broadening parameter $\gamma=0.025 \mathrm{eV}$. We solve Eq. (5) using a random-phase approximation, i.e. taking $K^{\mathrm{xc}}=0$. It was demonstrated $^{61}$ that for transition metals this is a rather good approximation for the description of low-energy electronic excitations, i.e., in an energy range of primary interest here.

\section{CALCULATION RESULTS AND DISCUSSION}

The calculated band structure as a basic ingredient of the dielectric matrix evaluation is shown for Pd in the vicinity of the Fermi level in Fig. 1. This band structure is in good agreement with previous theoretical studies ${ }^{62-66}$ although some small quantitative differences in energy positions of energy bands resulted from the use of different calculation methods can be noted. In the figure one can see how three energy bands cross the Fermi level. As will be shown below this produces strong effect on the low-energy response properties in palladium.

In Fig. 2 we present the calculated dielectric function for

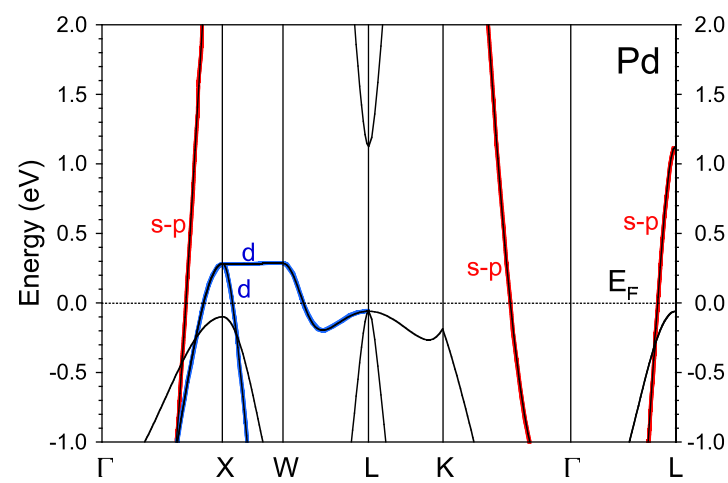

FIG. 1. (Color online) Calculated band structure of Pd in vicinity of the Fermi level, $E_{F}$, along some high-symmetry directions of the Brillouin zone. The energy bands crossing the Fermi level are highlighted by colors according to their main symmetry—red (gray) and blue (dark gray) colors stand for the $s-p$ and $d$ bands, respectively. The energies are according to the Fermi level shown by dotted horizontal line.

$\mathrm{Pd}, \epsilon(\mathbf{q}, \omega) \equiv \epsilon_{\mathrm{G}=0, \mathbf{G}^{\prime}=0}(\mathbf{q}, \omega)$, and imaginary part of inverse dielectric matrix, $\operatorname{Im}\left[\epsilon_{\mathbf{G}=0, \mathbf{G}^{\prime}=0}^{-1}(\mathbf{q}, \omega)\right]$-a so-called energyloss function - at $q=0.0712$ a.u. $^{-1}$ along the $\langle 100\rangle$ symmetry direction as a function of energy $\omega$ for small $\omega$. In the figure one can clearly see that in this energy region these quantities are qualitatively different from that calculated with the use of the FEG model. In particular, one can observe that $\operatorname{Re}[\epsilon]$ instead of one zero-crossing in this energy region (as it has $\operatorname{Re}[\epsilon]$ calculated within the FEG model) indeed has three such zero crossings. This is explained by the fact that there are two dominant peaks in $\operatorname{Im}[\epsilon]$ : a peak A centered at $\omega=0.09 \mathrm{eV}$ and a peak $\mathrm{C}$ at $\omega=0.54 \mathrm{eV} .{ }^{67} \mathrm{As} \operatorname{Im}[\epsilon]$ and $\operatorname{Re}[\epsilon]$ are interrelated via the Hilbert transformation, this makes $\operatorname{Re}[\epsilon]$ to reach zero three times. Whereas the zero

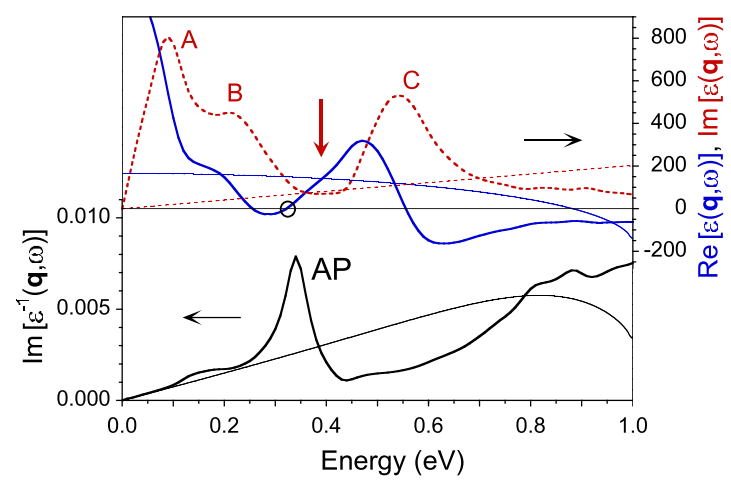

FIG. 2. (Color online) Top panel: real (solid line) and imaginary (dashed line) parts of dielectric function $\epsilon(\mathbf{q}, \omega)$ for Pd versus energy $\omega$ at the momentum transfer $|\mathbf{q}|=0.0712$ a.u. ${ }^{-1}$ along the $\langle 100\rangle$ symmetry direction. Low panel: the respective energy-loss function, $\operatorname{Im}\left[\epsilon^{-1}(\mathbf{q}, \omega)\right]$. In both panels thick lines present results of $a b$ initio calculations, whereas thin lines show the FEG model results obtained with the use of $r_{s}=3.5$ proposed for the description of the electronic system in Pd (Ref. 44). Circle highlights the energy where $\operatorname{Re}[\epsilon]$ has a zero crossing which, in combination with corresponding local minimum in $\operatorname{Im}[\epsilon]$ (shown by vertical arrow), leads to the appearance of the AP peak in the an initio energy-loss function, $\operatorname{Im}\left[\epsilon^{-1}(\mathbf{q}, \omega)\right]$, corresponding to an acoustic plasmon. 
crossings at 0.25 and $0.55 \mathrm{eV}$ do not produce any peak structure in the energy-loss function because of coincidence with the local peaks at fairly the same energies in the $\operatorname{Im}[\epsilon]$ part, the zero crossing of $\operatorname{Re}[\epsilon]$ at $0.32 \mathrm{eV}$ (highlighted in Fig. 2 by a circle) combined with a local minimum in $\operatorname{Im}[\epsilon]$ at the same energy does produce a sharp peak in $\operatorname{Im}\left[\epsilon^{-1}\right]$. The presence of this peak is a signature of existence of a well-defined collective mode at this energy. Similar calculations performed for other small momenta in all three symmetry directions demonstrate that the energy positions of local maxima $\mathrm{A}, \mathrm{B}$, and $\mathrm{C}$ in $\operatorname{Im}[\epsilon]$ and corresponding minima between them disperse quasilinearly with $|\mathbf{q}|$. This behavior is explained by the next observation: at small $|\mathbf{q}|$ the energy for intraband transitions in Eq. (7) can be expressed as

$$
\epsilon_{n \mathbf{k}+\mathbf{q}}-\epsilon_{n \mathbf{k}}=\frac{\epsilon_{n \mathbf{k}+\mathbf{q}}-\epsilon_{n \mathbf{k}}}{\mathbf{k}+\mathbf{q}-\mathbf{k}} \cdot \mathbf{q} \approx \mathbf{u}_{n \mathbf{k}} \cdot \mathbf{q},
$$

where $\mathbf{u}_{n \mathbf{k}}=\partial \boldsymbol{\epsilon}_{n \mathbf{k}} / \partial \mathbf{k}$ is the group velocity in band $n$ at a given wave vector $\mathbf{k}$. As the maximum number of such intraband transitions in a given band $n$ occurs with the $\mathbf{u}_{n \mathbf{k}}$ components close to its maximal value, the corresponding structure in $\operatorname{Im}[\epsilon]$, which is a measure of a number for electron-hole $(e-h)$ transitions, is peaked at the high-energy side of this $e-h$ region. ${ }^{1}$ As a result, the slope of dispersions of maxima in $\operatorname{Im}[\epsilon]$ is determined by the corresponding maximal Fermi velocity components in a given direction for each energy bands crossing the Fermi surface.

Figure 3 shows density of states of the energy bands at the Fermi level versus their energy and magnitude of the group velocity components. One can note significant difference between the group velocity components in the $s-p$ and $d$ bands. As the positions of peaks in $\operatorname{Im}[\epsilon]$ disperse fairly linearly with the magnitude of $q$, the same do the peaks, corresponding to the acoustic plasmon, in $\operatorname{Im}\left[\epsilon^{-1}\right]$. The collection of the peak positions in $\operatorname{Im}\left[\epsilon^{-1}\right]$ for transfer momenta in the three main symmetry directions in $\mathrm{Pd}$, is presented in Fig. 4. In the figure the dispersion of the acoustic plasmons is shown by solid lines, whereas the peak positions corresponding to interband transitions are presented by dashed lines. One can see that whereas along the $\langle 100\rangle$ and $\langle 111\rangle$ symmetry directions there exist only one branch corresponding to the acoustic plasmon, along the $\langle 110\rangle$ one there are two such branches. The reason for this is explained in Fig. 5 where dielectric $\epsilon(\mathbf{q}, \omega)$ and energy-loss $\operatorname{Im}\left[\epsilon^{-1}\right]$ functions are presented for momentum transfer $\mathbf{q}$ along the $\langle 110\rangle$ symmetry direction with $q=0.0504$ a.u. ${ }^{-1}$. Unlike Fig. 2, here one can observe three clear local maxima $\mathrm{A}, \mathrm{B}$, and $\mathrm{C}$ in $\operatorname{Im}[\epsilon]$ which are explained by the fact that along this direction the strong anisotropy of the group velocity components of all three bands crossing the Fermi level occurs. The presence of the three peaks in $\operatorname{Im}[\epsilon]$ in this energy interval leads to the appearance of five zero crossings in $\operatorname{Re}[\epsilon]$. As a result, the two of them (denoted by circles in Fig. 5) in combination with the local minima in $\operatorname{Im}[\epsilon]$ produce two clear $\mathrm{AP}_{1}$ and $\mathrm{AP}_{2}$ peaks in $\operatorname{Im}\left[\epsilon^{-1}\right]$ corresponding to two distinct acoustic plasmon modes. The calculated group velocities of acoustic plasmons in $\mathrm{Pd}$ for the three main symmetry directions are presented in Table I. One can see that these values are of two orders of magnitude higher than the sound velocity in metals. Hence
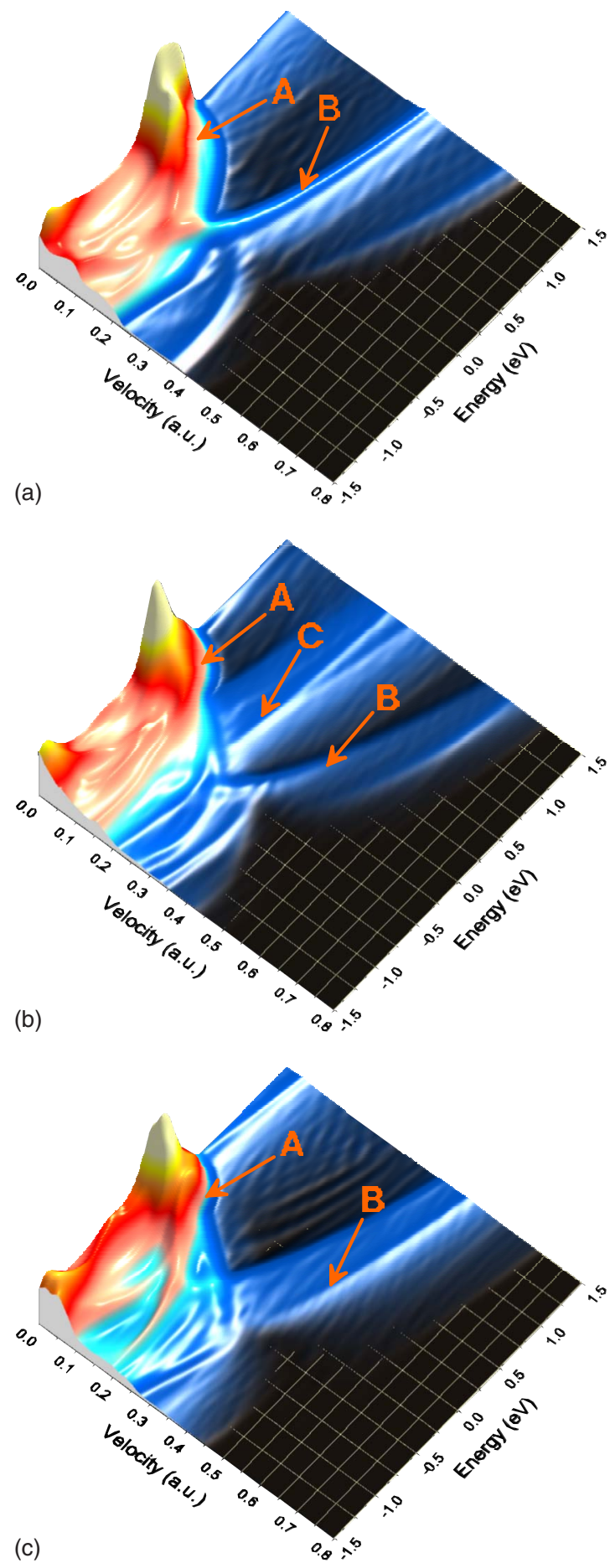

FIG. 3. (Color online) DOS of the energy bands in vicinity of the Fermi level in Pd versus its energy and group velocity components along three main symmetry directions: (a) $\langle 100\rangle$; (b) $\langle 110\rangle$; (c) $\langle 111\rangle$. Peaks denoted as A and C (B) correspond to DOS in $d$ $(s-p)$ bands. The energies are according to the Fermi level.

these acoustic plasmons in Pd can not interact directly with an acoustic phonon mode. ${ }^{25}$

Here we would like to stress the importance of achieving of convergency in the calculations in order to obtain the unambiguous results regarding the low-energy dielectric properties of real metals. In Fig. 6, as an example, we demon- 


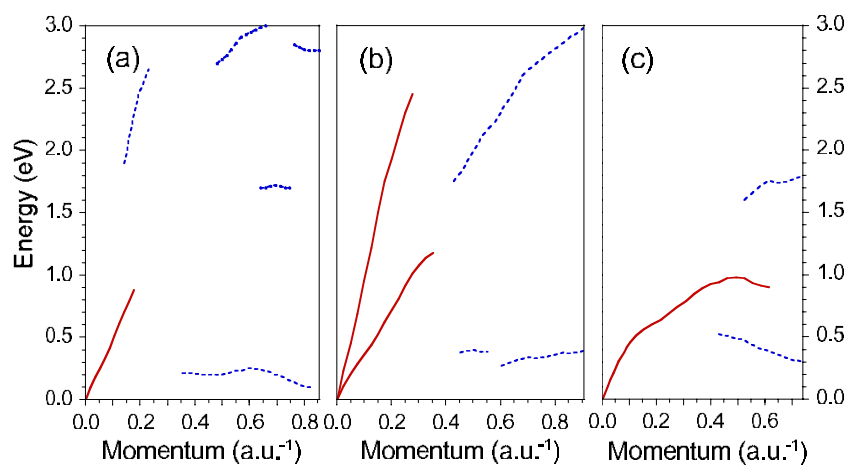

FIG. 4. (Color online) Dispersion of peaks in calculated $a b$ initio energy-loss function of Pd for the momentum transfer $\mathbf{q}$ along the (a) $\langle 100\rangle$, (b) $\langle 110\rangle$, and (c) $\langle 111\rangle$ symmetry directions. Acoustic-plasmon dispersions are depicted by solid lines, whereas dashed lines show dispersion of less-pronounced energy-loss peaks having its origin in interband transitions.

strate how the evaluated dielectric function at a small momentum transfer $\mathbf{q}$ crucially depends on value of the broadening parameter $\gamma$ employed in the evaluation of the spectral function via Eq. (7). Thus, at the smallest $\gamma$ value a well-defined AP peak corresponding to the acoustic plasmon appears at $\omega=0.18 \mathrm{eV}$. When the $\gamma$ value increases the energy of this peak is blueshifted and its width increases significantly. As $\gamma$ increases further more this mode even disappears. This is accompanied by dramatic changes in the dynamical dielectric function. Note that at large $\gamma$, instead of three zero crossings in this energy range, the $a b$ initio $\operatorname{Re}[\epsilon]$ part crosses zero only once and has behavior similar to that expected from the FEG model. In this respect quite instructive situation has occurred with the $\mathrm{MgB}_{2}$ compound. After discovery of superconductivity in this material, ${ }^{68}$ it was suggested on base of simple tight-binding calculations that an

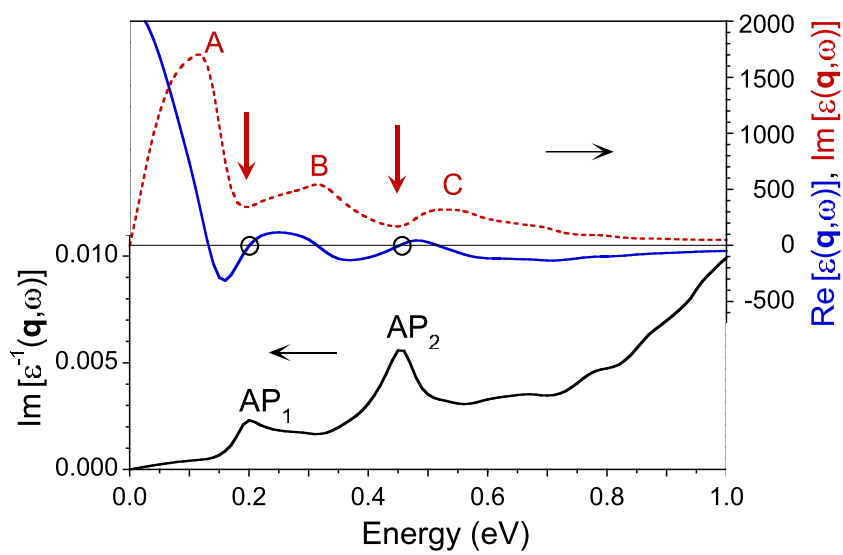

FIG. 5. (Color online) Top panel: real (solid line) and imaginary (dashed line) parts of dielectric function $\epsilon(\mathbf{q}, \omega)$ of Pd versus energy, $\omega$, for the momentum transfer $|\mathbf{q}|=0.0504$ a.u. $^{-1}$ along the $\langle 110\rangle$ symmetry direction. Low panel: the respective energy-loss function, $\operatorname{Im}\left[\epsilon^{-1}(\mathbf{q}, \omega)\right]$. Circles highlight energies where $\operatorname{Re}[\epsilon]$ has zero crossings, a fact which in combination with corresponding local minima in $\operatorname{Im}[\epsilon]$ (depicted by vertical arrows) leads to the appearance of peaks $\mathrm{AP}_{1}$ and $\mathrm{AP}_{2}$ in energy-loss function, corresponding to two acoustic plasmon modes.
TABLE I. Group velocities (in a.u.) of acoustic plasmons in Pd along three symmetry directions.

\begin{tabular}{lccc}
\hline \hline Direction & $\langle 100\rangle$ & $\langle 110\rangle$ & $\langle 111\rangle$ \\
\hline$u_{\mathrm{AP}}^{1}$ & 0.18 & 0.13 & 0.19 \\
$u_{\mathrm{AP}}^{2}$ & & 0.34 & \\
\hline \hline
\end{tabular}

acoustic plasmon could exist there. ${ }^{24}$ Nevertheless, subsequent $a b$ initio calculations ${ }^{69,70}$ did not confirm that claim. However, very recently this plasmon mode has been found in very detailed ab initio calculations..$^{26,27}$

The results presented here signal about paramount importance of achievement of convergency in the evaluation of the dynamical momentum-dependent dielectric function and consequently dynamical Coulomb interaction in real solids. In particular, it would be of great interest to investigate possible relation to the evaluated superconducting properties of materials within an "ab initio" approach ${ }^{71,72}$ where the dynamical Coulomb interaction is a key ingredient. Thus the difference of order of $10 \mathrm{~K}$ in the predicted $T_{c}$ in $\mathrm{MgB}_{2}$ between two calculations ${ }^{71,72}$ which used different approximations for the description of dynamical Coulomb interaction points out to the importance of inclusion of the proper dynamical screening.

This work presents for the first time an unambiguous $a b$ initio evidence of existence of an acoustic plasmon mode in an elemental 3D bulk metal such as Pd. Recent successful experimental observation in the energy-loss experiment ${ }^{73}$ of an analogous acoustic mode ${ }^{74,75}$ at a metal surface in a nice agreement with $a b$ initio predictions ${ }^{73}$ points out to the principal possibility of experimental detection of this kind of excitations in bulk as well. Regardless of a possible relevance of acoustic plasmons for superconductivity, ${ }^{76}$ the present findings amplify our basic knowledge of the low-

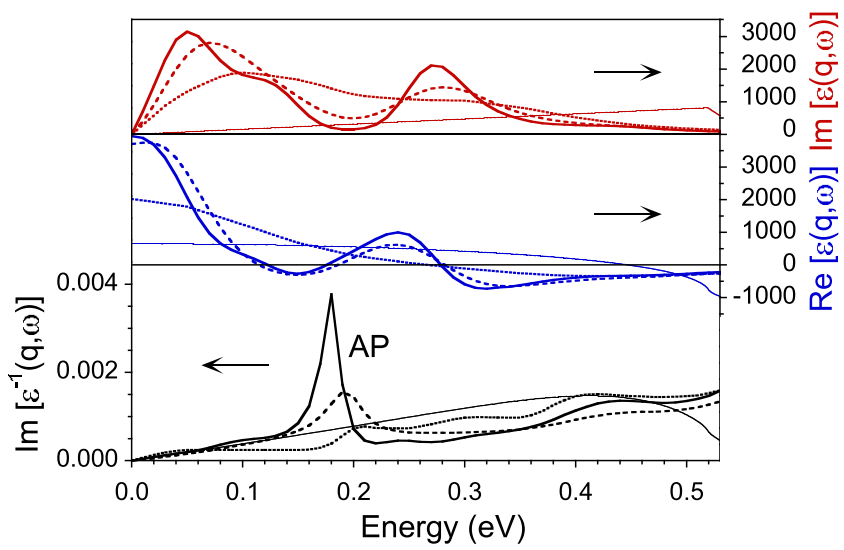

FIG. 6. (Color online) Dependence of imaginary (upper panel) and real (middle panel) parts of the dielectric function $\epsilon(\mathbf{q}, \omega)$, as well as the corresponding energy-loss function, $\operatorname{Im}\left[\epsilon^{-1}(\mathbf{q}, \omega)\right]$, on the broadening parameter $\gamma$ employed in the evaluation of the $S_{\mathbf{G}_{\mathbf{G}, \mathbf{G}^{\prime}}^{0}}(\mathbf{q}, \omega)$ matrix. Momentum $\mathbf{q}$ is along the $\langle 100\rangle$ symmetry direction with $|\mathbf{q}|=0.036$ a.u. $^{-1}$. Solid, dashed, and dotted lines stand for results obtained with $\gamma=0.025 \mathrm{eV}, \gamma=0.050 \mathrm{eV}$, and $\gamma$ $=0.100 \mathrm{eV}$, respectively. The corresponding FEG results, obtained with the use of $r_{s}=3.5$ a.u., are presented by thin solid lines. 
energy dynamical electronic screening properties of solids which can qualitatively differ from the expectations of simplified theories.

\section{CONCLUSIONS}

We have presented a theoretical study of electronic excitation spectra in Pd with the focus on the low-energy domain. The calculations were performed with the full inclusion of the electron band structure obtained within selfconsistent pseudopotential approach. We demonstrate that the presence in Pd of two kinds of carriers (in $s-p$ and $d$ bands) at the Fermi level produces dramatic modification of the excitation spectra at these energies in comparison with FEG model predictions. Thus at low energies, additionally to several well-defined features in the loss spectra due to numerous interband transitions in this metal, at small momenta a peculiar plasmon mode, resulting from the presence of fast (in the $s-p$ band) and slow (in the $d$ bands) carriers, with characteristic sound-like dispersion-acoustic plasmon-is predicted. This plasmon posses a strong directional dependence on momenta: whereas for momentum transfer along the $\langle 100\rangle$ and $\langle 111\rangle$ symmetry directions it arises as an single mode, along the $\langle 110\rangle$ symmetry direction two acoustic modes with different slopes appear.

Finally, our analysis points out that one should be very careful in performing such kind of evaluation. In particular one should pay special care regarding the convergence in the $\mathbf{k}$ sampling over the Brillouin zone in the evaluation of the density-response function $\chi^{o}$ of noninteracting electrons.

\section{ACKNOWLEDGMENTS}

We acknowledge the partial support from the University of the Basque Country (Grant No. GIC07IT36607), the Departamento de Educación del Gobierno Vasco, and the Spanish Ministerio de Ciencia y Tecnología (MCyT) (Grant No. FIS200766711C0101).
${ }^{1}$ D. Pines and P. Nozières, The Theory of Quantum Liquids, V. 1: Normal Fermi Liquids (W. A. Benjamin, New York, 1966).

${ }^{2}$ J. Lindhard, Mat. Fys. Medd. K. Dan. Vidensk. Selsk. 28, 1 (1954).

${ }^{3}$ K. Zeppenfeld, Z. Phys. 243, 229 (1971).

${ }^{4}$ M. G. Bell and W. Y. Liang, Adv. Phys. 25, 53 (1976).

${ }^{5}$ U. Büchner, Phys. Status Solidi B 81, 227 (1977).

${ }^{6}$ R. Ahuja, S. Auluck, J. M. Wills, M. Alouani, B. Johansson, and O. Eriksson, Phys. Rev. B 55, 4999 (1997).

${ }^{7}$ M. F. Lin, C. S. Huang, and D. S. Chuu, Phys. Rev. B 55, 13961 (1997).

${ }^{8}$ E. E. Krasovskii, W. Schattke, V. N. Strocov, and R. Claessen, Phys. Rev. B 66, 235403 (2002).

${ }^{9}$ R. Schuster, R. Kraus, M. Knupfer, H. Berger, and B. Büchner, Phys. Rev. B 79, 045134 (2009).

${ }^{10}$ A. A. Lucas, L. Henrard, and Ph. Lambin, Phys. Rev. B 49, 2888 (1994).

${ }^{11}$ M. F. Lin and Kenneth W.-K. Shung, Phys. Rev. B 50, 17744 (1994).

${ }^{12}$ T. Pichler, M. Knupfer, M. S. Golden, J. Fink, A. Rinzler, and R. E. Smalley, Phys. Rev. Lett. 80, 4729 (1998).

${ }^{13}$ H. Ehrenreich and H. R. Philipp, Phys. Rev. 128, 1622 (1962).

${ }^{14}$ R. M. Morgan and D. W. Lynch, Phys. Rev. 172, 628 (1968).

${ }^{15}$ M. A. Cazalilla, J. S. Dolado, A. Rubio, and P. M. Echenique, Phys. Rev. B 61, 8033 (2000).

${ }^{16}$ A. vom Felde, J. Sprösser-Prou, and J. Fink, Phys. Rev. B 40, 10181 (1989).

${ }^{17}$ F. Aryasetiawan and K. Karlsson, Phys. Rev. Lett. 73, 1679 (1994).

${ }^{18}$ D. Pines, Can. J. Phys. 34, 1379 (1956).

${ }^{19}$ V. Radhakrishnan, Phys. Lett. 16, 247 (1965).

${ }^{20}$ B. T. Geilikman, Sov. Phys. JETP 21, 796 (1965).

${ }^{21}$ H. Fröhlich, J. Phys. C 1, 544 (1968).

${ }^{22}$ D. A. Papaconstantopoulos, Handbook of the Band Structure of Elemental Solids (Plenum Press, New York, 1986).

${ }^{23}$ J. Ruvalds, Adv. Phys. 30, 677 (1981).
${ }^{24}$ K. Voelker, V. I. Anisimov, and T. M. Rice, arXiv:cond-mat/ 0103082 (unpublished).

${ }^{25}$ R. F. Wood, B. E. Sernelius, and A. L. Chernyshev, Phys. Rev. B 66, 014513 (2002).

${ }^{26}$ V. M. Silkin, A. Balassis, P. M. Echenique, and E. V. Chulkov, Phys. Rev. B 80, 054521 (2009).

${ }^{27}$ A. Balassis, E. V. Chulkov, P. M. Echenique, and V. M. Silkin, Phys. Rev. B 78, 224502 (2008).

${ }^{28}$ V. Z. Kresin and H. Morawitz, Phys. Rev. B 37, 7854 (1988).

${ }^{29}$ A. Bill, H. Morawitz, and V. Z. Kresin, Phys. Rev. B 66, 100501(R) (2002).

${ }^{30}$ A. Bill, H. Morawitz, and V. Z. Kresin, Phys. Rev. B 68, 144519 (2003).

${ }^{31}$ V. Z. Kresin and S. A. Wolf, Rev. Mod. Phys. 81, 481 (2009).

${ }^{32} \mathrm{We}$ would like to point out that we call this mode "acoustic plasmon" in the sense that its dispersion is linearly proportional to its momentum, i.e., it is similar to the dispersion of acoustic phonon branch in solids. The slope of acoustic plasmon dispersion can be notably steeper as the Fermi velocity of carriers in a "slow" band, $u_{F}^{\text {slow }}$ can exceed substantially the velocity of sound in solids. However, analyzing the band structures of metals (Ref. 22) in vicinity of the Fermi surface one can note that indeed $v_{F}^{\text {slow }}$ can vary in a rather wide interval, especially in transition metals.

${ }^{33}$ P. A. Bennett and J. C. Fuggle, Phys. Rev. B 26, 6030 (1982).

${ }^{34}$ F. P. Netzer and M. M. El Gomati, Surf. Sci. 124, 26 (1983).

${ }^{35}$ G. Chiarello, E. Colavita, M. De Crescenzi, and S. Nannarone, Phys. Rev. B 29, 4878 (1984).

${ }^{36}$ M. Nishijima, M. Jo, Y. Kuwahara, and M. Onchi, Solid State Commun. 58, 75 (1986).

${ }^{37}$ T. Bornemann, J. Eickmans, and A. Otto, Solid State Commun. 65, 381 (1988).

${ }^{38}$ H. A. E. Hagelin, J. F. Weaver, G. B. Hoflund, and G. N. Salaita, J. Electron Spectrosc. Relat. Phenom. 124, 1 (2002) and references therein.

${ }^{39}$ J. H. Weaver, Phys. Rev. B 11, 1416 (1975). 
${ }^{40}$ Handbook of Optical Constants of Solids, edited by E. D. Palik (Academic Press, San Diego, 1991).

${ }^{41}$ E. G. Maksimov, I. I. Mazin, S. N. Rashkeev, and Yu. A. Uspenski, J. Phys. F: Met. Phys. 18, 833 (1988).

${ }^{42}$ G.-M. Fehrenbach, Phys. Rev. B 59, 15085 (1999).

${ }^{43}$ E. E. Krasovskii and W. Schattke, Phys. Rev. B 63, 235112 (2001).

${ }^{44}$ V. P. Zhukov, F. Aryasetiawan, E. V. Chulkov, and P. M. Echenique, Phys. Rev. B 65, 115116 (2002).

${ }^{45}$ V. J. Keast, J. Electron Spectrosc. Relat. Phenom. 143, 97 (2005).

${ }^{46}$ V. M. Silkin, I. P. Chernov, P. M. Echenique, Yu. M. Koroteev, and E. V. Chulkov, Phys. Rev. B 76, 245105 (2007).

${ }^{47}$ I. P. Chernov, Yu. M. Koroteev, V. M. Silkin, and Yu. I. Tyurin, Dokl. Akad. Nauk 420, 758 (2008) [Dokl. Phys. 53, 318 (2008)].

${ }^{48}$ R. Lässer and N. V. Smith, Phys. Rev. B 25, 806 (1982).

${ }^{49}$ E. Runge and E. K. U. Gross, Phys. Rev. Lett. 52, 997 (1984).

${ }^{50}$ M. Petersilka, U. J. Gossmann, and E. K. U. Gross, Phys. Rev. Lett. 76, 1212 (1996).

${ }^{51}$ I. G. Gurtubay, W. Ku, J. M. Pitarke, and A. G. Eguiluz, Comput. Mater. Sci. 30, 104 (2004).

${ }^{52}$ W. Ku and A. G. Eguiluz, Phys. Rev. Lett. 82, 2350 (1999).

${ }^{53}$ N. E. Maddocks, R. W. Godby, and R. J. Needs, Europhys. Lett. 27, 681 (1994).

${ }^{54}$ E. E. Krasovskii and W. Schattke, Phys. Rev. B 60, R16251 (1999).

${ }^{55}$ F. Aryasetiawan and O. Gunnarsson, Phys. Rev. B 49, 16214 (1994).

${ }^{56}$ F. Aryasetiawan, in Strong Coulomb Correlations in Electronic Structure Calculations, edited by V. I. Anisimov (Gordon and Beach, Singapore, 2001).

${ }^{57}$ D. M. Ceperley and B. J. Alder, Phys. Rev. Lett. 45, 566 (1980); as parametrized by J. P. Perdew and A. Zunger, Phys. Rev. B 23, 5048 (1981).

${ }^{58}$ N. Troullier and J. L. Martins, Phys. Rev. B 43, 1993 (1991).

${ }^{59}$ S. L. Adler, Phys. Rev. 126, 413 (1962).

${ }^{60}$ N. Wiser, Phys. Rev. 129, 62 (1963).

${ }^{61}$ I. G. Gurtubay, J. M. Pitarke, W. Ku, A. G. Eguiluz, B. C. Larson, J. Tischler, P. Zschack, and K. D. Finkelstein, Phys. Rev.
B 72, 125117 (2005)

${ }^{62}$ N. V. Smith, Phys. Rev. B 9, 1365 (1974).

${ }^{63}$ N. E. Christensen, Phys. Rev. B 14, 3446 (1976).

${ }^{64}$ C. T. Chan and S. G. Louie, Phys. Rev. B 27, 3325 (1983).

${ }^{65}$ H. Chen, N. E. Brener, and J. Callaway, Phys. Rev. B 40, 1443 (1989).

${ }^{66}$ I. Yu. Sklyadneva, A. Leonardo, P. M. Echenique, S. V. Eremeev, and E. V. Chulkov, J. Phys.: Condens. Matter 18, 7923 (2006).

${ }^{67}$ Note that at this $\mathbf{q}$, the peak B in $\operatorname{Im}[\epsilon(\mathbf{q}, \omega)]$ is too weak in order to have any significance, although as it is shown below the corresponding peak for the momentum transfers q's along the $\langle 110\rangle$ direction is much more pronounced producing an additional acoustic plasmon mode.

${ }^{68}$ J. Nagamatsu, N. Nakagawa, T. Muranaka, Y. Zenitani, and J. Akimitsu, Nature (London) 410, 63 (2001).

${ }^{69}$ V. P. Zhukov, V. M. Silkin, E. V. Chulkov, and P. M. Echenique, Phys. Rev. B 64, 180507(R) (2001).

${ }^{70}$ W. Ku, W. E. Pickett, R. T. Scalettar, and A. G. Eguiluz, Phys. Rev. Lett. 88, 057001 (2002).

${ }^{71}$ A. Floris, G. Profeta, N. N. Lathiotakis, M. Lüders, M. A. L. Marques, C. Franchini, E. K. U. Gross, A. Continenza, and S. Massidda, Phys. Rev. Lett. 94, 037004 (2005).

${ }^{72}$ A. Floris, A. Sanna, M. Lüders, G. Profeta, N. N. Lathiotakis, M. A. L. Marques, C. Franchini, E. K. U. Gross, A. Continenza, and S. Massidda, Physica C 456, 45 (2007).

${ }^{73}$ B. Diaconescu, K. Pohl, L. Vattuone, L. Savio, Ph. Hofmann, V. M. Silkin, J. M. Pitarke, E. V. Chulkov, P. M. Echenique, D. Farías, and M. Rocca, Nature (London) 448, 57 (2007).

${ }^{74}$ V. M. Silkin, A. García-Lekue, J. M. Pitarke, E. V. Chulkov, E. Zaremba, and P. M. Echenique, Europhys. Lett. 66, 260 (2004).

${ }^{75}$ V. M. Silkin, J. M. Pitarke, E. V. Chulkov, and P. M. Echenique, Phys. Rev. B 72, 115435 (2005).

${ }^{76}$ From Fig. 4 and Table I it is clear that acoustic plasmons in Pd have too high group velocities to induce any effect on superconductivity in this material. This is because the large value of the group velocity reduces the phase space for virtual transitions involving acoustic plasmons, leading to a small value of the coupling constant for the pairing and to inability to overcome direct static Coulomb repulsion. This is in accord with absence of superconductivity in this metal. 